Artículo

\title{
Variables de influencia para la producción de miel utilizando abejas Apis mellifera en la región de Misantla
}

Anett Yeli Martell-Tamanis

Fabiola Guadalupe Lobato-Rosales

Margarito Landa-Zárate

Giovanni Luna-Chontal

Luis Enrique García-Santamaría

Gregorio Fernández-Lambert ${ }^{\S}$

Instituto Tecnológico Superior de Misantla. Carretera a Loma del Cojolite km 1.8, Misantla, Veracruz, México. CP. 93821. (172T0857@itsm.edu.mx; 172T0855@itsm.edu.mx; mlandaz@itsm.edu.mx; glunac@itsm.edu.mx; legarcias@itsm.edu.mx).

${ }^{\S}$ Autor para correspondencia: gfernandezl@itsm.edu.mx.

\section{Resumen}

Diversas notas de portales electrónicos, documentales y en menor grado artículos de corte científico, han reportado el interés por mejorar el rendimiento de producción de miel por colmena. Entre estos trabajos pueden encontrarse reportados, en su forma dimensional, factores climáticos, sociales y tecnológicos, en donde expertos y especialistas reconocen que contribuyen a mejorar la producción de miel. Apoyado de expertos apícolas, del método Delphi y el análisis estructural MICMAC, en este artículo se reporta la influencia y dependencia de 22 variables y su relación directa e indirecta con la producción de miel en la región intermunicipal de Misantla del distrito apícola núm. 52. Las variables con base en MICMAC se clasifican en clave, reguladoras, palanca y autónomas. En el plano estratégico, la 'ubicación del apiario' relacionado con la 'diversidad de floración melífera' y el 'tiempo que transcurre entre la colocación del apiario y la cosecha de miel', representan las variables clave con mayor valor estratégico de 75/79, 61/79 y $63 / 79$, respectivamente. Si bien, la 'floración de especies melíferas' es una variable clave para producción de miel, el resultado también indica que esta variable no es una determinante para que el apicultor decida la 'ubicación de apiarios'. Estos resultados aportan conocimiento en cuatro categorías de variables influyentes en la producción de miel para que el apicultor reflexione sobre sus practica apícolas y pueda diseñar estrategias y tomar decisiones, para mejorar el rendimiento de producción miel.

Palabras clave: apiario, colmena, MICMAC, miel de abeja, producción apícola.

Recibido: julio de 2019

Aceptado: septiembre de 2019 


\section{Introducción}

La apicultura en México es una actividad relevante del subsector pecuario, con volumen de producción que la ubican en el sexto lugar a nivel mundial y en América se posiciona en tercer lugar en producción y exportación (Magaña et al., 2016). Esta actividad representa la tercera fuente generadora de divisas en el sector agropecuario en México (Ulloa et al., 2010; Luna et al., 2016). La Secretaría de Agricultura, Ganadería, Desarrollo rural, Pesca y Alimentación (SAGARPA) divide a la República Mexicana en cinco regiones apícolas, con diferentes grados de desarrollo y variedad de tipos de mieles de acuerdo a sus características de humedad, color, aroma y sabor. Estas regiones son: Altiplano, Pacífico, Golfo, Norte y Península de Yucatán.

La región apícola en el estado de Veracruz se encuentra ubicada en la región del Golfo. De acuerdo con el Servicio de Información Agroalimentaria y Pesquera del Gobierno de México (SIAP), dicho estado ocupa el tercer lugar como productor de miel a nivel nacional, con $4704091 \mathrm{t}$ año ${ }^{-1}$, lo que representa 9.21\% a nivel nacional, con valor estimado de 2190411 millones de pesos. El estado de Veracruz cuenta con 12 distritos apícolas, dentro de los cuales, el distrito núm. 52 Martínez de la Torre lo conforman 22 municipios y en este distrito destacan como productores de miel, los municipios de Papantla, Martínez de la Torre, Tlapacoyan, San Rafael y Misantla.

El municipio de Misantla se ubica en la región montañosa central del estado de Veracruz, sobre la vertiente del Golfo de México, entre la sierra Madre Oriental y la Sierra de Chiconquiaco, colindando con los municipios de Yecuatla, Colipa, Nautla, Tenochtitlan, Martínez de la Torre, Atzalan y Vega de Alatorre.

Misantla presenta características naturales que favorecen el desarrollo de la apicultura, como son los tipos de climas: tropical cálido, cálidos subhúmedos, templados húmedos y de régimen térmico cálido; con precipitaciones que varían de los $2036.4 \mathrm{~mm}$ anuales en promedio y temperatura media anual que oscila entre los $22.7{ }^{\circ} \mathrm{C}$ con humedad relativa de $35 \%$ a $40 \%$. Asimismo, el tipo de vegetación como chalahuite (Inga vera) y los cultivos intensivos de café (Coffea arabica), toronja (Citrus maxima), Lima persa (Citrus latifolia Tanaka), mandarina (Citrus reticulata), son componentes de flora natural en esta región.

En consecuencia, la miel que se produce en la región intermunicipal de Misantla acorde al tipo de floración son la miel de cítrico y miel multiflora, con subproductos como el polen, la cera, y el propóleo. Cuando menos $85 \%$ de los apicultores de la región de estudio realizan la explotación tradicional enfocada a la producción de miel, cera y núcleos (Luna et al., 2016), contraponiéndose con la denominada explotación integral que busca obtener ingresos adicionales a partir de la obtención de otros productos como jalea real, apitoxina, mieles mono-florales, miel orgánica, además de servicios de polinización.

Luna et al. (2016) menciona que los esquemas de la actividad apícola en México se catalogan en tres grupos: tecnificado, semi tecnificado y tradicional, de los cuales, en la región intermunicipal de Misantla 9\% de los productores apícolas son tecnificados, 36\% son semi tecnificados y $45 \%$ usan el método tradicional. En este contexto, Misantla se mantiene posicionado en el quinto lugar. Con base a datos del SIAP, la producción promedio de este municipio es de 80 toneladas anuales que representa $6.26 \%$ de la producción total del distrito y $1.71 \%$ de Veracruz. 
La apicultura en la región de Misantla enfrenta un panorama de oportunidades no sólo de tipo económico e infraestructura, si no de conocimiento relacionado con los factores que influyen en la producción de miel. En este sentido Magaña et al. (2016) mencionan que la productividad de miel es resultado de la conjugación de varios factores, entre los que figuran la tecnología y el ambiente físico natural.

Así también, Abou-Shaara et al. (2013) indica que factores como la densidad de la flora melífera y netarífera y minimizar el recorrido de la abeja en su pecoreo influyen en los rendimientos. Magaña et al. (2016) añade que la productividad de miel por colmena se encuentra asociada a diversos factores o eventos fisíco naturales, entre los cuales estan la africanización de las colonias, la deforestación de selvas y bosques, los huracanes y recientemente el cambio climático.

En este sentido, Medina-Cuellar et al. (2014) reportan como factores de la variabilidad en la producción de miel a las fluctuaciones climáticas las cuales impactan en la fenología de las plantas, y en consecuencia la fuente de néctar y comportamiento de las abejas. Mientras que, Roque et al. (2016) reportan como factores para mejorar la producción de miel, los factores topográficos, de clima, la densidad de floración, así como la ubicación del apiario, aunado a los factores no controlables como la temperatura, la humedad relativa, tipo de suelo, viento, luz solar, entre otros, dentro de los que pueden mencionarse la preferencia de la abeja a ciertas especies florales, debido a la cantidad del néctar producido y la accesibilidad a la fuente de alimento, así como a la composición del polen.

Si bien estos estudios reportan factores dimensionales en la tecnología, el clima y la topografía en la que se asientan los apiarios, éstos carecen de un análisis de la influencia dependencia de las variables que conforman estas dimensiones. Este artículo presenta un estudio que utiliza el análisis estructural para determinar la influencia y dependencia entre variables en los factores climáticos, topográficos, económicos, tecnológicos y de trashumancia en la región intermunicipal de Misantla. En este sentido, también se aporta una categorización de las variables de acuerdo con la dimensión de las variables analizadas por el análisis estructural.

\section{Materiales y métodos}

Se realiza una investigación de tipo exploratoria soportada en fuentes primarias y secundarias. La información primaria se recoge mediante entrevistas no estructuradas, cara a cara y un cuestionario diagnóstico con técnicos y expertos en el sector apícola, productores de miel, representantes y ex representantes de asociaciones de apicultores de la región de estudio.

De una población de 52 socios en la Asociación de Apicultores de Misantla (AAM), se entrevistaron 30 apicultores, mientras que aquellos no registrados en la AAM, se identificaron mediante la técnica de bola de nieve lineal durante el periodo enero a mayo del año 2018. La región de estudio comprende la zona centro-norte del Estado de Veracruz, delimitada a los municipios de Misantla (19 $9^{\circ} 55^{\prime} 51.86$ ' latitud norte $96^{\circ} 51^{\prime} 6.09$ longitud oeste); Yecuatla (19 $9^{\circ} 51^{\prime}$ 57' latitud norte $96^{\circ} 46^{\prime} 36^{\prime}$ ' longitud oeste; Colipa (19 $55^{\prime}$ ' $25^{\prime}$ ' latitud norte $96^{\circ} 43^{\prime} 38^{\prime}$ ' longitud oeste; Juchique (19 $50^{\prime}$ ' $25^{\prime}$ ' latitud norte $96^{\circ} 41^{\prime}$ '41' 'longitud oeste) y Tenochtitlan (19 48' $27^{\circ}$ ' latitud norte $96^{\circ} 54^{\prime}$ 39' 'longitud oeste). La colindancia de estos municipios cubre un área 1159 km², con una población aproximada de 65996 habitantes. 
Para esta investigación se utiliza el análisis estructural de Godet y Durance (2011) avalado por la Organización de las Naciones Unidas para la Educación, la Ciencia y la Cultura (UNESCO) para estudios de prospectiva y análisis estructural a nivel internacional (Martínez, 2012). Esta metodología es comúnmente utilizada como una herramienta de estructuración y reflexión colectiva, así como para generar información estratégica para la toma de decisiones, el diseño, elaboración e implementación de actividades de mejora continua en los sistemas de trabajo con la posibilidad de describir un sistema con ayuda de una matriz que relaciona todos sus elementos constitutivos (Godet y Durance, 2007; Ballesteros y Ballesteros, 2008; Quintero y López, 2010; Garza y Cortez, 2011).

El análisis estructural es la primera fase de la metodología de escenarios y su importancia radica en que permite evidenciar de forma clara las relaciones existentes entre las variables que caracterizan el sistema de estudio (Cely, 1999). Este análisis descrito en la Figura 1, comprende tres fases del método MICMAC como matriz de impactos cruzados-multiplicación aplicada a una clasificación (Godet y Durance, 2007).

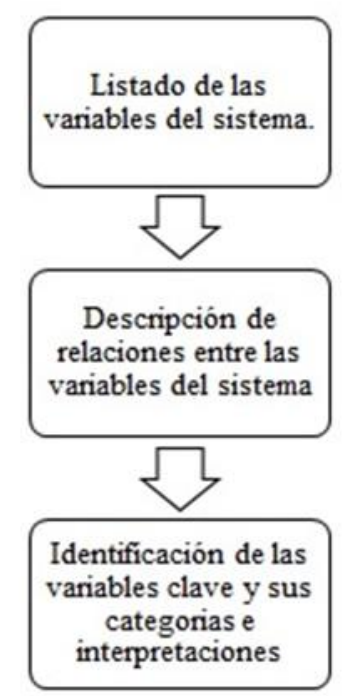

Figura 1. Fases de la metodología de análisis estructural. Fuente: Godet y Durance (2007).

En la primera fase, se acude a personas con experiencia demostrada y vigente como apicultor y por medio de entrevistas abiertas, se seleccionan y definen las variables que influyen en el sistema de producción de miel. En esta fase, de acuerdo con criterios de trayectoria-tiempo de apicultor, sistema de trabajo apícola demostrado por el apicultor y tamaño del productor apícola, se integra un panel de expertos con cinco productores de miel.

En la segunda fase, apoyado de un panel de expertos, se construye la matriz estructural para evaluar la influencia que ejerce cada una de las variables del sistema de producción entre sí. El panel de expertos asigna un valor de acuerdo al grado de influencia: nula (0); débil (1); media (2); y fuerte (3). Posteriormente, se procesan estas interrelaciones mediante el software MICMAC. Como se muestra en la Figura 2, la resultante del cálculo de la matriz estructural es la clasificación de las variables de acuerdo con la ubicación en un plano de influencia-dependencia. El Cuadro A1, detalla la interpretación de cada una de estas variables en el sistema de producción. 
La tercera fase consiste en identificar las variables clave, su influencia y su dependencia. En primer lugar, mediante una clasificación directa, y finalmente, por una clasificación indirecta.

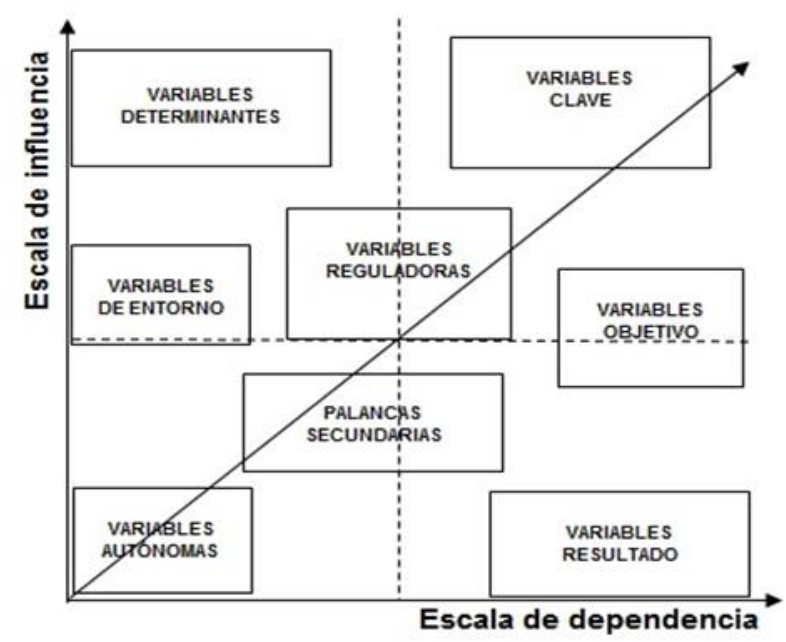

Figura 2. Plano estratégico de influencias-dependencias de doble entrada MICMAC. Fuente: Ambrosio et al. (2011).

\section{Resultados y discusión}

\section{Fase I}

Mediante entrevista abierta cara-cara con apicultores se recogen las variables que influyen en la producción de miel, y en consenso con el panel de expertos, se identifican y definen 22 variables. El Cuadro 1 describe 22 variables en un título largo y un título corto. Este último se utiliza en el programa MICMAC.

Cuadro 1. Variables que afectan la producción de miel.

\begin{tabular}{|c|c|c|c|}
\hline Núm. & Título largo & Título corto & Descripción \\
\hline $\mathrm{I}$ & $\begin{array}{l}\text { Tiempo dedicado a la } \\
\text { actividad }\end{array}$ & Tiempo (Ti) & $\begin{array}{l}\text { Tiempo que le dedica el apicultor a la } \\
\text { actividad, puede ser medio tiempo o tiempo } \\
\text { completo. }\end{array}$ \\
\hline 2 & $\begin{array}{l}\text { Deforestación de } \\
\text { especies apícolas }\end{array}$ & $\begin{array}{l}\text { Deforesta } \\
\text { (D) }\end{array}$ & $\begin{array}{l}\text { Aumento de la deforestación de especies } \\
\text { melíferas que son de importancia para la } \\
\text { apicultura. }\end{array}$ \\
\hline 3 & Uso de pesticidas & $\begin{array}{l}\text { Pesticidas } \\
\quad(\mathrm{P})\end{array}$ & $\begin{array}{l}\text { Uso de pesticidas e insecticidas en sitios de } \\
\text { importancia apícola o en las zonas de pecoreo. }\end{array}$ \\
\hline 4 & $\begin{array}{l}\text { Aumento de zonas } \\
\text { agropecuarias }\end{array}$ & $\begin{array}{l}\text { Agropecuar } \\
\text { (A) }\end{array}$ & $\begin{array}{l}\text { Aumento de áreas dedicadas a la ganadería y } \\
\text { zonas de cultivos no aptos para la apicultura. }\end{array}$ \\
\hline 5 & $\begin{array}{l}\text { Crecimiento } \\
\text { poblacional }\end{array}$ & $\begin{array}{l}\text { Población } \\
\qquad(\mathrm{Po})\end{array}$ & $\begin{array}{l}\text { Aumento de la mancha urbana en zonas aptas } \\
\text { para la apicultura. }\end{array}$ \\
\hline
\end{tabular}




\begin{tabular}{|c|c|c|c|}
\hline Núm. & Título largo & Título corto & Descripción \\
\hline 6 & $\begin{array}{l}\text { Fenología de especies } \\
\text { melíferas }\end{array}$ & $\begin{array}{l}\text { Fenología } \\
\quad(\mathrm{Fe})\end{array}$ & $\begin{array}{l}\text { Fenología de las especies melíferas aptas para } \\
\text { la apicultura. }\end{array}$ \\
\hline 7 & $\begin{array}{l}\text { Temperaturas elevadas } \\
\text { dentro de la colmena }\end{array}$ & $\begin{array}{l}\text { Tempera } \\
\quad(\mathrm{Te})\end{array}$ & $\begin{array}{l}\text { Temperatura mayor a } 35{ }^{\circ} \mathrm{C} \text { dentro de la } \\
\text { colmena. }\end{array}$ \\
\hline 8 & $\begin{array}{l}\text { Exceso de humedad } \\
\text { dentro de la colmena }\end{array}$ & $\begin{array}{l}\text { Humedad } \\
\qquad(\mathrm{H})\end{array}$ & $\begin{array}{l}\text { Exceso de humedad mayor a } 18 \% \text { dentro de la } \\
\text { colmena. }\end{array}$ \\
\hline 9 & $\begin{array}{l}\text { Acceso a zonas de } \\
\text { floración }\end{array}$ & Acceso (Ac) & Accesibilidad a las zonas de floración. \\
\hline 10 & $\begin{array}{l}\text { Distancia a zonas de } \\
\text { floración }\end{array}$ & $\begin{array}{l}\text { Dist-flora } \\
\text { (Df) }\end{array}$ & $\begin{array}{l}\text { Distancia que existe entre la zona de floración } \\
\text { y el apiario. }\end{array}$ \\
\hline 11 & $\begin{array}{l}\text { Distancia entre } \\
\text { apiarios }\end{array}$ & $\begin{array}{l}\text { Dist-apia } \\
\quad \text { (Da) }\end{array}$ & Distancia que existe entre cada apiario. \\
\hline 12 & $\begin{array}{l}\text { Exceso de lluvia en la } \\
\text { región productora }\end{array}$ & Lluvia (Ll) & $\begin{array}{l}\text { Exceso de lluvia que no permita el pecoreo o } \\
\text { que lave el néctar de las flores. }\end{array}$ \\
\hline 13 & Edad de la abeja reina & Edad $(\mathrm{E})$ & $\begin{array}{l}\text { Edad en la que es considerada productiva la } \\
\text { abeja reina. }\end{array}$ \\
\hline 14 & $\begin{array}{l}\text { Morfología de las } \\
\text { especies melíferas }\end{array}$ & $\begin{array}{l}\text { Morfología } \\
\text { (M) }\end{array}$ & $\begin{array}{l}\text { Estructura y forma de algunas especies } \\
\text { melíferas, las cuales no son aptas para la } \\
\text { recolección de néctar. }\end{array}$ \\
\hline 15 & Robo de colmenas & Robo (Ro) & $\begin{array}{l}\text { Robo de colmenas en apiarios o durante la } \\
\text { trashumancia. }\end{array}$ \\
\hline 16 & $\begin{array}{l}\text { Ubicación de sitios } \\
\text { aptos }\end{array}$ & $\begin{array}{l}\text { Ubicación } \\
\text { (U) }\end{array}$ & Ubicación de zonas aptas de pecoreo. \\
\hline 17 & $\begin{array}{l}\text { Depredadores } \\
\text { potenciales }\end{array}$ & $\begin{array}{l}\text { Depredador } \\
\text { (De) }\end{array}$ & $\begin{array}{l}\text { Especies animales que son consumidores de } \\
\text { miel o de abejas. }\end{array}$ \\
\hline 18 & $\begin{array}{l}\text { Cuidados durante } \\
\text { trashumancia }\end{array}$ & Cuidados (C) & $\begin{array}{l}\text { Medidas preventivas que se les brinda a las } \\
\text { colmenas durante la trashumancia. }\end{array}$ \\
\hline 19 & Revisiones periódicas & $\begin{array}{l}\text { Revisión } \\
\quad(\mathrm{Re})\end{array}$ & $\begin{array}{l}\text { Lapso de tiempo que se brinda para la revisión } \\
\text { de las colmenas, las cuales pueden ser diarias, } \\
\text { semanales, quincenales. }\end{array}$ \\
\hline 20 & Tiempo de cosecha & $\begin{array}{l}\text { T-cosecha } \\
\quad(\mathrm{Tc})\end{array}$ & $\begin{array}{l}\text { Tiempo que transcurre entre la colocación de } \\
\text { apiario y la cosecha. }\end{array}$ \\
\hline 21 & $\begin{array}{l}\text { Trashumancia a otras } \\
\text { regiones }\end{array}$ & $\begin{array}{l}\text { Trashuman } \\
\quad \text { (Tr) }\end{array}$ & $\begin{array}{l}\text { Trashumancia de las colmenas a zonas aptas } \\
\text { para la producción de miel. }\end{array}$ \\
\hline 22 & $\begin{array}{l}\text { Floración de especies } \\
\text { melíferas }\end{array}$ & $\begin{array}{l}\text { Floración } \\
\text { (Fl) }\end{array}$ & $\begin{array}{l}\text { Floración de especies melíferas que son } \\
\text { consideradas potenciales para la apicultura. }\end{array}$ \\
\hline
\end{tabular}

Fuente: elaboración a partir de MICMAC en Ambrosio et al. (2011). 


\section{Fase II}

En la Fase II, se construye una matriz de información de doble entrada, en la cual se fijan las variables que fueron seleccionadas por el panel de expertos, utilizando el método Delphi, se ejecutan rondas de consulta entre los expertos los días 28, 29, 30 y 01 de octubre del año 2018, para identificar la influencia de las variables descritas en la matriz de información. Por cada pareja de variables se planteó la siguiente pregunta: ¿existe una relación de influencia directa entre la variable $i$ y la variable $j$ ? ¿de existir dicha influencia, en qué grado existe? (Pérez y Alfonso, 2016). Los expertos evalúan la influencia directa entre todas las variables y su intensidad, de la siguiente manera: fuerte (3); media (2); débil (1); y nula (0) o potencial (P).

Con base a Del Río y Cárdenas (2018), la matriz estructural, descrita en la Figura 3, se introduce en la base de datos del MICMAC. Esta matriz se entiende de la siguiente manera: la humedad (H) mantiene una 'fuerte' influencia con la temperatura (Te) y así, sucesivamente. La evaluación de la matriz por medio del MICMAC se refleja en el plano cartesiano de influencias/dependencias, mediante dos clasificaciones: directas e indirectas.

\begin{tabular}{|c|c|c|c|c|c|c|c|c|c|c|c|c|c|c|c|c|c|c|c|c|c|c|}
\hline a & $\bar{z}$ & $\begin{array}{l}\stackrel{\sim}{\theta} \\
\ddot{2}\end{array}$ & $\underset{\ddot{v}}{\omega}$ & $\ddot{\Rightarrow}$ & $\dddot{\leftrightarrow}$ & $\frac{a}{\ddot{\sigma}}$ & $\stackrel{\ddot{\sigma}}{\ddot{\theta}}$ & $\begin{array}{l}\infty \\
\ddot{I}\end{array}$ & $\stackrel{\circ}{\ddot{z}}$ & $\begin{array}{l}\check{0} \\
\ddot{g}\end{array}$ & $=$ & $\begin{array}{l}\bar{N} \\
\ddot{E}\end{array}$ & $\begin{array}{l}\omega \\
\ddot{m}\end{array}$ & $\begin{array}{l}\bar{s} \\
\dot{s}\end{array}$ & $\begin{array}{l}\overline{\tilde{\sigma}} \\
\bar{\sigma}\end{array}$ & $\begin{array}{l}\bar{a} \\
\ddot{c}\end{array}$ & $\underset{\ddot{\theta}}{\ddot{\sigma}}$ & $\begin{array}{l}\bar{\infty} \\
\ddot{\sigma}\end{array}$ & $\begin{array}{l}\bar{\zeta} \\
\ddot{\sigma}\end{array}$ & $\begin{array}{l}\tilde{o} \\
\ddot{a} \\
\vec{a}\end{array}$ & $\begin{array}{l}\underline{N} \\
\ddot{g}\end{array}$ & $\begin{array}{l}N \\
\because \\
\Xi\end{array}$ \\
\hline $1: \mathrm{Ti}$ & 0 & 0 & 0 & 0 & 0 & 3 & 0 & 0 & 0 & 0 & 0 & 0 & 0 & 0 & 2 & 3 & 3 & 1 & 3 & 1 & 3 & 0 \\
\hline $2: D$ & 0 & 0 & 2 & 3 & 3 & 0 & 0 & 0 & 0 & 3 & 3 & 0 & 0 & 0 & 0 & 3 & 0 & 0 & 0 & 3 & 3 & 3 \\
\hline $3: \mathrm{P}$ & 0 & 2 & 0 & 3 & 1 & 0 & 0 & 0 & 0 & 1 & 1 & 0 & 0 & 0 & 0 & 3 & 0 & 0 & 0 & 0 & 2 & 3 \\
\hline $4: A$ & 0 & 3 & 3 & 0 & 0 & 0 & 0 & 0 & 1 & 2 & 3 & 0 & 0 & 0 & 0 & 3 & 0 & 0 & 0 & 0 & 2 & 3 \\
\hline $5: \mathrm{Po}$ & 0 & 3 & 1 & 0 & 0 & 0 & 0 & 0 & 0 & 0 & 1 & 0 & 0 & 0 & 0 & 3 & 0 & 0 & 0 & 0 & 2 & 3 \\
\hline $6: \mathrm{Fe}$ & 0 & 0 & 0 & 0 & 0 & 0 & 0 & 0 & 0 & 0 & 0 & 0 & 0 & 0 & 0 & 3 & 0 & 0 & 2 & 0 & 3 & 3 \\
\hline $7: \mathrm{Te}$ & 0 & 0 & 0 & 0 & 0 & 0 & 0 & 3 & 0 & 0 & 0 & 1 & 0 & 0 & 0 & 0 & 0 & 1 & 0 & 0 & 0 & 0 \\
\hline $8: H$ & 0 & 0 & 0 & 0 & 0 & 0 & 3 & 0 & 0 & 0 & 0 & 2 & 0 & 0 & 0 & 0 & 3 & 1 & 0 & 0 & 0 & 0 \\
\hline $9: A c$ & 0 & 1 & 0 & 0 & 0 & 3 & 0 & 0 & 0 & 3 & 0 & 2 & 0 & 0 & 0 & 3 & 0 & 0 & 3 & 2 & 1 & 2 \\
\hline $10: \mathrm{Df}$ & 0 & 2 & 2 & 2 & 0 & 0 & 0 & 0 & 3 & 0 & 3 & 0 & 0 & 0 & 0 & 3 & 0 & 0 & 0 & 3 & 0 & 2 \\
\hline $11: \mathrm{Da}$ & 0 & 0 & 0 & 2 & 0 & 0 & 0 & 0 & 0 & 0 & 0 & 0 & 0 & 0 & 0 & 3 & 0 & 0 & 0 & 2 & 0 & 0 \\
\hline $12: \mathrm{Ll}$ & 0 & 0 & 0 & 0 & 0 & 2 & 0 & 2 & 0 & 0 & 0 & 0 & 0 & 0 & 0 & 0 & 1 & 1 & 0 & 2 & 0 & 2 \\
\hline $13: E$ & 0 & 0 & 0 & 0 & 0 & 0 & 0 & 0 & 0 & 0 & 0 & 0 & 0 & 0 & 0 & 0 & 0 & 0 & 0 & 0 & 0 & 0 \\
\hline $14: \mathrm{M}$ & 0 & 0 & 0 & 0 & 0 & 0 & 0 & 0 & 0 & 0 & 0 & 0 & 0 & 0 & 0 & 1 & 0 & 0 & 0 & 0 & 0 & 0 \\
\hline $15:$ Ro & 2 & 0 & 0 & 0 & 0 & 0 & 0 & 0 & 0 & 0 & 0 & 0 & 0 & 0 & 0 & 0 & 0 & 3 & 2 & 0 & 0 & 0 \\
\hline $16: U$ & 0 & 3 & 3 & 3 & 3 & 3 & 0 & 0 & 3 & 3 & 3 & 0 & 0 & 1 & 3 & 0 & 0 & 0 & 0 & 3 & 3 & 3 \\
\hline $17: \mathrm{De}$ & 0 & 0 & 0 & 0 & 0 & 0 & 0 & 3 & 0 & 0 & 0 & 0 & 0 & 0 & 0 & 1 & 0 & 0 & 1 & 0 & 0 & 0 \\
\hline $18: C$ & 2 & 0 & 0 & 0 & 0 & 0 & 0 & 0 & 0 & 0 & 0 & 0 & 0 & 0 & 2 & 0 & 1 & 0 & 0 & 0 & 0 & 0 \\
\hline $19: \operatorname{Re}$ & 2 & 0 & 0 & 3 & 0 & 0 & 0 & 0 & 3 & 0 & 0 & 0 & 0 & 0 & 1 & 1 & 1 & 0 & 0 & 0 & 1 & 0 \\
\hline $20: \mathrm{Tc}$ & 3 & 3 & 0 & 0 & 0 & 3 & 0 & 1 & 2 & 3 & 3 & 3 & 2 & 0 & 3 & 3 & 2 & 2 & 2 & 0 & 3 & 3 \\
\hline $21: \operatorname{Tr}$ & 2 & 1 & 0 & 3 & 3 & 2 & 0 & 0 & 3 & 0 & 0 & 0 & 0 & 0 & 2 & 2 & 0 & 3 & 0 & 3 & 0 & 2 \\
\hline $22: \mathrm{F} 1$ & 0 & 3 & 3 & 3 & 0 & 3 & 0 & 0 & 3 & 3 & 3 & 2 & 0 & 0 & 0 & 3 & 0 & 0 & 0 & 3 & 3 & 0 \\
\hline
\end{tabular}

Figura 3. Matriz estructural MICMAC. Fuente: Elaboración con apoyo del programa MICMAC Godet y Durance (2007).

La primera clasificación se realiza mediante las sumas de valores de influencia/dependencia para cada una de las variables y la segunda clasificación es mediante la aplicación del método MICMAC por medio de la elevación de la matriz a la segunda potencia, existiendo una correlación entre las variables floración de especies melíferas y ubicación de zonas aptas de 1520 interacciones. 
La Figura 4 muestra el plano de influencias-dependencias directas resultante de la matriz de impactos cruzados, producto del nivel de motricidad y dependencia entre variables. La motricidad y dependencia de las variables depende de la ubicación de las variables dentro del plano de influencia-dependencia. Las variables motrices son las que ejercen mayor influencia sobre el resto de las variables que componen el sistema. Las variables dependientes, son aquellas que están influenciadas por el resto de las variables (Vázquez et al., 2017).

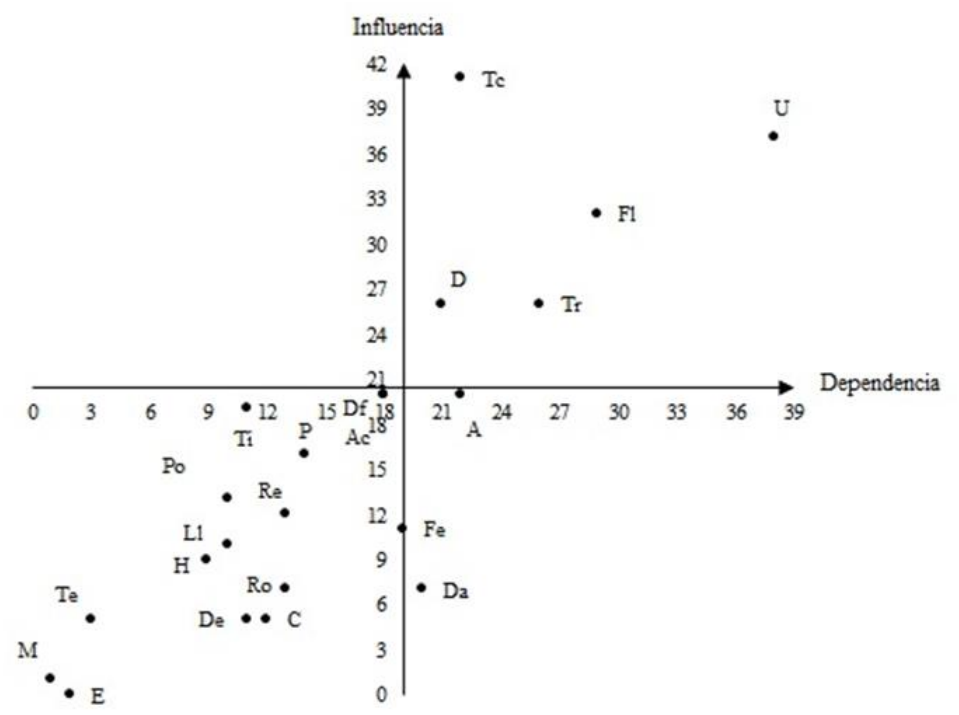

Figura 4. Plano de influencias-dependencias. Fuente: elaboración con apoyo del programa MICMAC Godet y Durance (2007).

Como se ha descrito en la Figura 4, MICMAC realiza una clasificación de las variables que conforman el sistema con base en su ubicación en el plano de influencias-dependencias. El resultado de esta clasificación fue la siguiente.

Variables clave: ubicación de zonas aptas de pecoreo, floración de especies melíferas, tiempo de cosecha.

Variables reguladoras: trashumancia, deforestación.

Palancas secundarias: tiempo dedicado a la actividad, distancia a zonas de floración, acceso a zonas de floración, aumento de zonas agropecuarias, uso de pesticidas, fenología de especies melíferas, distancia entre apiarios, revisiones periódicas.

Variables autónomas: robo de colmenas, exceso de lluvia en la región productora, crecimiento poblacional, cuidados por transporte de la colmena durante trashumancia, exceso de humedad dentro de la colmena, temperatura elevada dentro de la colmena, depredadores potenciales, morfología de las especies melíferas, edad de la abeja reina.

La clasificación de las variables es un proceso que permitió identificar la importancia de las variables y su carácter estratégico en la producción de miel. El valor estratégico de las variables indica la relevancia que la variable tiene en la producción de miel y en ese grado, el cuidado y atención que debería guardar el apicultor en su cadena-producto. En un sentido amplio, actuar sobre ellas, conlleva efectos de evolución del resto de las variables y en consecuencia del sistema apícola de la región de estudio. 
En el Cuadro 2 se describe la clasificación de las variables resultado del plano de influenciadependencia directa y su valor estratégico como una correlación de la magnitud de influencia en la producción de miel y dado que no se identificaron en el plano variables determinantes, variables de entorno, variables objetivo y variables resultado, se omiten sus columnas en la Cuadro 2. En donde, el valor estratégico $\left(\mathrm{E}_{\mathrm{n}}\right)$ de la variable está determinado por la suma de su motricidad $\left(\mathrm{m}_{\mathrm{n}}\right)$ y de su valor de dependencia $\left(\mathrm{d}_{\mathrm{n}}\right)$.

Cuadro 2. Clasificación y valor estratégico de variables de influencia-dependencia en la producción de miel.

\begin{tabular}{|c|c|c|c|c|c|c|c|}
\hline \multicolumn{2}{|c|}{ Reguladora } & \multicolumn{2}{|c|}{ Palancas secundarias } & \multicolumn{2}{|c|}{ Clave } & \multicolumn{2}{|c|}{ Autónoma } \\
\hline Variable & $E_{n}$ & Variable & $E_{n}$ & Variable & $\mathrm{E}_{\mathrm{n}}$ & Variable & $E_{n}$ \\
\hline $\mathrm{D}$ & 47 & Ac & 38 & Tc & 63 & Ro & 20 \\
\hline $\operatorname{Tr}$ & 52 & A & 42 & $\mathrm{Fl}$ & 61 & $\mathrm{Ll}$ & 20 \\
\hline & & $\mathrm{P}$ & 30 & $\mathrm{U}$ & 75 & Po & 23 \\
\hline & & $\mathrm{Fe}$ & 30 & & & $\mathrm{C}$ & 17 \\
\hline & & $\mathrm{Da}$ & 27 & & & $\mathrm{H}$ & 18 \\
\hline & & $\mathrm{Re}$ & 25 & & & $\mathrm{Te}$ & 8 \\
\hline & & Df & 38 & & & De & 16 \\
\hline & & $\mathrm{Ti}$ & 30 & & & M & 2 \\
\hline & & & & & & $\mathrm{E}$ & 2 \\
\hline
\end{tabular}

Fuente: elaboración con apoyo del panel de expertos y Godet y Durance (2007).

La Figura 5, muestra la influencia directa entre variables proporcionada por el programa MICMAC Cruz y Medina (2015), en la cual se observan las relaciones entre variables conectadas con arcos, los cuales están identificados con la magnitud de la influencia. Por ejemplo, con influencia débil (1); influencia media (2); influencia importante; y (3).

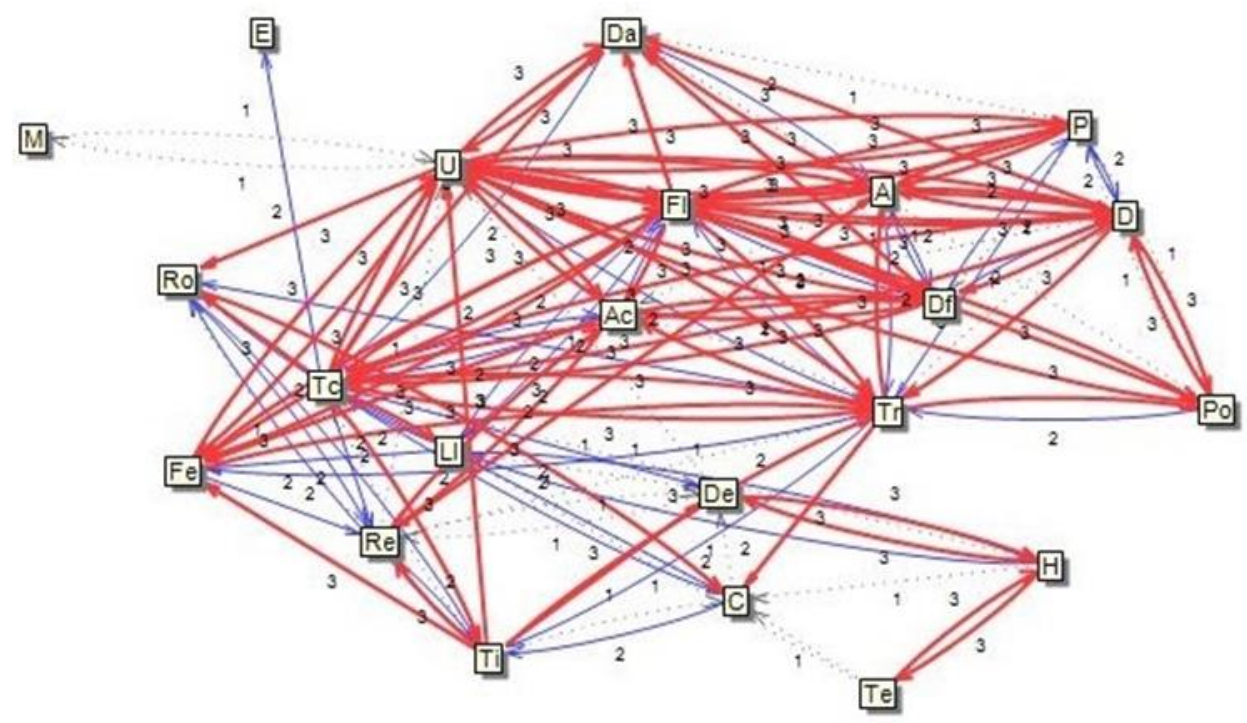

Figura 5. Gráfico de influencias directas. Fuente: elaboración con apoyo del programa MICMAC Godet y Durance (2007). 
Puede observarse que las variables con mayor interacción e influencia dentro del sistema, en orden de su valor estratégico, son: ubicación de zonas aptas de pecoreo (U_75), tiempo de cosecha (Tc_ 63), floración de especies melíferas (Fl_61), trashumancia (Tr_52), deforestación de especies apícolas (D_47), aumento de zonas agropecuarias (A_42), distancia a zonas de floración(Df_38), acceso a zonas de floración (Ac_38), fenología de especies melíferas (Fe_30), distancia entre apiarios (Da_27), y el crecimiento poblacional (Po_23).

De esta forma, el programa MICMAC genera un diagrama de influencias indirectas, en el cual se observan las variables con un grado de influencia indirectamente importante en la producción de miel. En este diagrama se observa que la 'floración de especies melíferas' mantiene una influencia indirecta importante con la 'ubicación de zonas aptas de pecoreo' para la práctica apícola. Esto resulta interesante, ya que, estas variables se identifican como variables con mayor interacción e influencia e importantes para la producción de miel en el diagrama de influencias directas.

Esto hace ver que, si bien la 'floración de especies melíferas' es importante para producción de miel, esta variable no es una determinante en la ubicación de apiarios. Sin embargo, existe una influencia importante negativa de la 'deforestación de especies melíferas', el 'uso de pesticidas' y el 'aumento de zonas agropecuarias' con la actividad de 'trashumancia'.

Este hallazgo, es coincidente con lo reportado por Bellarby et al. (2008); Roque et al. (2016) y donde menciona que la trashumancia esta estrechamente ligada al ambiente y recursos naturales de la zona que el productor utiliza para instalar sus apiarios, las cuales han sido modificadas en la última década por diferentes factores, entre ellos, la tala clandestina o el incremento de extensión de cultivos intensivos, que modifican los sistemas bióticos con impactos en bajos rendimientos de producción de miel por colmena.

Es importante resaltar que dentro de las 22 variables reportadas en este artículo, el panel de expertos no considera el acceso a fuentes hídricas como una variable que preocupe al apicultor para incrementar la producción de miel en la colmena. Esta consideración la justifica el panel de expertos debido a la bondad de la zona de estudio en cuanto a que la colmena tiene acceso ilimitado a fuentes de agua. Sin embargo, en esta investigación hemos encontrado que la 'fuente hídrica' tiene una importancia de $27 \%$, entre los criterios de acceso al apiario, la altura sobre el nivel del mar y la temperatura de la zona, para la 'ubicación de apiarios' y para incrementar la producción de miel, respectivamente.

En este contexto, la investigación de campo deja ver que la 'selección del lugar del nectario', la 'selección de zonas de la flora melífera' y el 'transporte de la colmena al lugar de asentamiento del apiario', son actividades primarias que deben siempre cuidarse en la práctica de la apicultura para mejorar el rendimiento de producción de miel en la colmena.

Las dos primeras actividades son consideradas como variables claves en esta investigación con valor estratégico de 75 y 61 respectivamente en una escala máxima de 79, mientras que la actividad del 'transporte de la colmena al lugar de asentamiento del apiario', se considera en este estudio como una variable autónoma con valor estratégico de 17, la cual, aun y cuando con su bajo grado estratégico, ésta puede interferir negativamente en la producción de miel, en función del grado de su presencia. 
En congruencia a estos resultados, Simone et al. (2016); Alger et al. (2018) hacen énfasis que la movilidad de las colmenas, entre otros factores climatológicos y de prácticas agrícolas tradicionales como la aplicación de pesticidas, son elementos que estresan a la colmena con un efecto en el detrimento de la producción de miel.

Si bien en el plano de influencia-dependencia se sugieren tres variables clave y dos variables reguladoras con valor estratégico de 42/79 a 75/79 unidades estratégicas, debe tenerse en cuenta que el conjunto de variables de Palanca Secundarias son las variables que actúan sobre las variables reguladoras y éstas a su vez afecta la evolución de las variables clave. En sentido estricto, en coincidencia con Contreras-Uc et al. (2018), el rendimiento de producción de miel, depende en gran medida con de las prácticas apícolas integradas a la oportunidad y eficacia en el manejo de la colonia.

En un orden de importancia estratégica, las variables 'aumento de zonas dedicadas a la ganadería y zonas de cultivos no aptos para la apicultura' tiene una influencia importante indirecta con 'accesibilidad a las zonas de floración' y la 'distancia que existe entre la zona de floración y el apiario'. Los apicultores de la región de estudio advierten que el efecto de estas variables no se manifiesta de forma inmediata y su comportamiento, tarde o temprano, en mayor o menor grado, impactará en el nivel de floración necesaria para que la colmena realice su trabajo de pecoreo.

Finalmente, los hallazgos identificadas en la Fase I de esta investigación relacionados con la practica apicola, son coincidentes en gran medida con lo reportado por Contreras-Uc et al. (2018), como lo son la edad avanzada de los apicultores, la actividad no tecnificada para la producción de miel y que la apicultura es una forma completaría de ingreso económico. Esto hace suponer que existe una relación de la edad del apicultor con la adopción de procesos innovadores en la práctica apícola, toda vez que, con base a los resultados en este estudio, estos componentes pueden conjugarse para detonar las variables clave y variables con valor estratégico para mejorar el rendimiento de miel.

\section{Conclusiones}

La herramienta de análisis estructural permitió determinar y analizar la influencia y dependencia de variables entendidas como actividades que comúnmente el apicultor realiza como práctica en la producción de miel y que en la literatura sólo se reportan en la dimensión de factores que afectan la producción de miel de abeja. Los resultados muestran que las variables aquí reportadas, no sólo de manera directa, si no, que también de manera indirecta, influyen en la producción de miel desde la actividad clave de encontrar zonas aptas para el asentamiento de apiarios para cubrir las necesidades de proveeduría de néctar o polen de la colmena.

En tal forma, la selección del lugar del nectario, la selección de la zona de la flora melífera y su accesibilidad y transporte de la colmena al lugar de asentamiento del apiario, estratégicamente representan decisiones del apicultor, las cuales están bajo su control. En este sentido, consideramos relevante en este artículo reportar las variables que influyen en la producción de miel, así mismo, el grado de influencia/dependencia de las variables reportadas con el propósito de mejorar la compresión del sistema de producción de miel de abeja. 
En esta investigación se han utilizado el método Delphi y MICMAC con el propósito de reducir el sesgo de la subjetividad de los expertos para determinar e identificar la influencia de variables en la producción de miel. Este conocimiento ayudará al apicultor a definir sus acciones y decisiones, así como diseñar estrategias para mejorar el rendimiento de producción miel. Con este objetivo, merece atención como trabajos futuros estudios orientados a determinar criterios de ubicación y tamaños de apiarios que maximicen la producción de miel.

\section{Agradecimiento}

Por las facilidades e información proporcionada, los autores extienden su agradecimiento a los expertos que colaboraron con esta investigación. Especialmente Anett Yeli Martell Tamanis y Fabiola Lobato Rosales, agradecen al Consejo Nacional de Ciencia y Tecnología (CONACYT) por el apoyo de la beca de estudios 707840 y 707859 otorgada de febrero 2018 a agosto 2019, para realizar los estudios de Maestría en Ingeniería Industrial en el ITS de Misantla.

\section{Literatura citada}

Abou, S. H. F.; Al, G. A. A. and Mohamed, A. A. 2013. A suitability map for keeping honey bees under harsh environmental conditions using geographical information system. Arabia Saudita. World Appl. Sci. J. 22(8):1099-1105. DOI: 10.5829/idosi.wasj.2013.22.08.7384.

Alger, S. A.; Burnham, P. A.; Lamas, Z. S.; Brody, A. K. and Richardson, L. L. 2018. Home sick: impacts of migratory beekeeping on honey bee (Apis mellifera) pests, pathogens, and colony size. PeerJ, Inc. November 2. PubMed 30405967. http://doi.org/10.7717/peerj.5812.

Ambrosio, A. M.; Martín, L. J. M. y Pérez, H. P. P. 2011. Aplicación del análisis estructural de prospectiva al diseño de estrategias de desarrollo rural: el caso de la Comarca de Jeréz. España. Estudios de Economía Aplicada. 1(29):247-278.

Ballesteros, R. D. P. y Ballesteros, S. P. P. 2008. Análisis estructural prospectivo aplicado al sistema logístico. Scientia et Technica. 39(14):194-199.

Bellarby, J.; Foereid, B.; Hastings, A. and Smith, P. 2008. Cool farming: climate impacts of agriculture and mitigation potential. In: Campaigning for Sustainable Agriculture. (Ed.). greenpeace.org. JN 102. Greenpeace International. The Netherlands. 20-25 p.

Cely, B. A. V. 1999. Metodología de los escenarios para estudios prospectivos. Revista Ingeniería e Investigación. Issue 44:26-35. DOI: 10.15446/ing.investig.

Contreras, U. L. C.; Magaña, M. M. A y Sanginés, G. J. R. 2018. Características técnicas y socioeconómicas de la apicultura en comunidades mayas del Litoral Centro de Yucatán. México. Acta Universitaria. 28(1):77-86.

Cruz, A. P. L. y Medina, V. J. E. 2015. Selección de los métodos para la construcción de los escenarios de futuro. Colombia. Entramado. 1(11):32-46.

Del Río, C. A. A. y Cárdenas, Q. B. G. 2018. Dinámica de sistemas una forma de optimizar la gestión del riesgo. Revista Escuela de Administración de Negocios. Edición especial. 125$143 \mathrm{pp}$.

Garza, V. J. B. y Cortez, A. D. V. 2011. El uso del método MICMAC y MACTOR análisis prospectivo en un área operativa a través del Lean Manufacturing. México. Innovaciones de Negocios. 8(16):335-356.

Godet, M. y Durance, P. 2007. Prospectiva estratégica: problemas y métodos. Segunda edición Español. París. Donostia. San Sebastian. 104 p. 
Godet, M. y Durance, P. 2011. La prospectiva estratégica para las empresas y los territorios. Español, Karel García Cortina. Dunot. Tercera (Ed.). París. Colección Topos. 10. 142 p.

Luna, C. G.; Roque, P. J. G.; Fernández, L. G.; Tiburcio, A. I. y Landa, Z. M. 2016. Trashumancia apícola en la región de Misantla, Veracruz. México. In: Congreso interdiciplinario de ingenierías. 143-148 pp.

Magaña, M. M. A.; Tavera, C. M. E.; Salazar, B. L. L. y Sanginés, G. J. R. 2016. Productividad de la apicultura en México y su impacto sobre la rentabilidad. Rev. Mex. Cienc. Agríc. 5(7):1103-1115.

Martínez, M. M. 2012. Nuevos fundamentos en la investigación científica: computacionales, prospectiva y análisis estructural con el método MIC-MAC. (Ed.). Trillas. 1. México. 216 p.

Medina, C. S.; Portillo, V. M.; García, Á. C. J.; Terrazas, G. G. y Alba, N. L. 2014. Influencia del ambiente sobre la productividad de la segunda cosecha de miel de abeja en Aguascalientes de 1998 a 2010. México. Rev. Chapingo Ser. Cienc. Forest. Amb. 2(20):159-165.

Pérez, U. R. I. y Alfonso, V. H. 2016. El uso del método MICMAC, para la definición de procesos de intervención en las organizaciones. Colombia. Ciencia y Poder Aéreo. 1(11):92-105.

Quintero, P. D. A. y López, M. S. M. 2010. Análisis estructural: un apoyo para el modelado con dinámica de sistemas. Colombia. Avances en Sistemas e Informática. 3(7):153-161.

Roque, P. J. G., Luna, C. G.; Fernández, L. G.; Tiburcio, A. I. y Hernández, G. T. A. 2016. Enfoque de estudio territorial apoyado de sistemas de información geográfica para definir territorios apícolas en Misantla. Veracruz-México. In: Congreso Interdiciplinario de Ingenierias. 22-26 pp.

Simone, F. M.; Li, B. H.; Huang, M. H.; Strand, M. K.; Rueppell, O. and Tarpy, D. R. 2016. Migratory management and environmental conditions affect lifespan and oxidative stress in honey bees. London. Scientific Reports. 6:1-10. DOI:10.1038/srep32023.

Ulloa, J. A.; Mondragón, C. P. M.; Rodríguez, R. R.; Reséndiz, V. J. A. y Rosas, U. P. 2010. La miel de abeja y su importancia. México. Revista Fuente. Año 2(4):11-18.

Vázquez, A. S.; Luján, A. C.; Olivas, G. J. M.; González, H. H. G. y Luján, A. H. 2017. Prospectiva del sistema organizacional del sector forestal en Chihuahua, México. México. Madera y Bosques. 2(23):205-222. 